\title{
Editorial
}

\section{Fluorescence-Guided Surgery: The Quest for Precision}

\author{
Amr Mohsen \\ Professor of Surgery, Cairo University, Egypt
}

The ever-mounting pressure on surgeons to improve efficacy, safety and financial outcomes of their work, has driven them, and the industry, to seek ways for enhancing operative precision. We certainly need to delineate, beyond doubt, lesions that are supposed to be completely removed, as well as structures that ought to be preserved. One way to achieve this goal is to augment visualization at surgery using fluorescence techniques. The concept of fluorescence-guided surgery is based on making the target tissue shine by light emanating from within, thus standing out in the operative field more prominently, while the surgeon is actually at work, i.e., offering real-time augmented visualization.

Simply put, the principle is based on preferentially delivering a fluorescent material, a fluorophore, to the target tissue, followed by exposing the operative field to excitation light of a particular wavelength that lies in the invisible spectrum. Excitation of the fluorophore results in emission of a different light, a phenomenon that is called fluorescence. Emitted light is either visible and is picked up by the naked eye, or invisible and requires a special camera to see.

During the last decade, the surgical literature has witnessed a persistently growing number of publications addressing this evolving technology. Most of them have investigated the use of the fluorophore indocyanine green (ICG), which is excited by near-infrared (NIR) light. Popularity of this light is due to its tissue penetration to a depth that exceeds the conventional white light as it may reach down to $1 \mathrm{~cm}$. It, thus, allows the real-time vision of the operator to reach beneath the surface, albeit for no more than a centimetre. Two other fluorophores are in common use. These are fluorescein and 5-aminolevulinic acid (5-ALA).

The technology made its best impact in the field of cancer surgery. In this field ensuring complete cancer excision, meanwhile reducing collateral damage is the sine qua non for success. Success with making cancers fluoresce is based on the fact that the leaky nature of tumour vessels allows a fluorophore to gain a higher concentration in its tissues. Among the different types of studied malignancies, two have shown that fluorescence guidance is of notable value. Removal of intracranial gliomas under 5-ALA guidance has demonstrated that the real extension of the tumour is likely to be beyond that identified by MRI (1). Equally good, several research articles have attested to the added value of ICG/NIR for precisely locating liver tumours, intraoperatively (2). Furthermore, boosting precision of cancer localization can be achieved by selective targeting where the fluorophore is bound to a molecule that has avidity for certain neoplastic tissue. The first clinical example of this refinement was published in 2013 where conjugating folate with fluorescein allowed van Dam et al. very clear visualization of ovarian cancer deposits in the peritoneal cavity (3).

The new technology has also found its way for locating sentinel lymph nodes (SLN) in cases of breast cancer and melanoma surgery. Case series studies proved its feasibility and safety. It was also studied for accuracy either in its own or in combination with other modalities. A 2014 randomized controlled trial (4) compared blue dye with fluorescence technique for breast cancer SLN where ICG/NIR fluorescence proved to give a significantly higher detection rate. 
Outside the field of cancer surgery, the list of operative fluorescence guidance is long, yet two examples of popular operations are worth mentioning, namely cholecystectomy and intestinal resection. While intraoperative cholangiography (IOC) is the standard method for delineating bile duct anatomy at cholecystectomy, the technique is not perfect. It involves ionizing radiation and entails exhausting mental work in order to adapt the radiographic image to what the surgeon actually sees on a laparoscopy screen. Consequently, such adaptation is commonly inaccurate. Fluorescence guidance promises to fill this gap with its safety and real-time augmented visualization of the ducts. Multiple studies verified the efficacy and safety of ICG/NIR for ductal delineation. Others concluded that it could shorten operative time (5) while being easier to use and is less costly than IOC (6). Further less expensive and simpler fluorescent technology using fluorescein and ultraviolet light has more recently evolved. As the emitted light is within the visible spectrum it requires no special cameras to detect. The technique proved to be $100 \%$ specific and $82.5 \%$ sensitive for locating bile duct (7).

While fluorescence-guided cholecystectomy is new, fluorescence for assessing intestinal viability dates back to the mid 20th century. An intravenously administered fluorophore will reach vascularized segments and will fluoresce under excitation light while non-vascularized segments will not. The technique would, thus, guide the surgeon to the extent of resection and helps judge viability of an anastomosis. With the recent technological advances, the use of this principle has been revived, particularly with robotic resection of rectal cancer, a site that is known for a notoriously high leakage rate. Initial reports revealed encouraging results $(8,9)$.

Fluorescence-guided surgery suffers two weak points. The first is technical, which is limited penetration of the excitation light. Some may argue that this point might not be a significant obstacle because the surgeon will bring the tissue of interest closer to the surface during dissection. A more practical solution is combining fluorescence with another technique, e.g., ultrasound, Magnetic Resonance Imaging (MRI) or Positron Emission Tomography (PET) scan. The other Journal of Surgical Academia 2016;6(1):1-3 weak point that actually slows down its routine use in surgical practice is that most of the relevant research is not of high evidence level. In fact, only a few randomized controlled trials were published. This is clearly a problem that will be solved with time, as more high-quality research will be generated.

The advantages of this technology certainly outnumber its disadvantages. The main one is the real-time augmentation of the surgeon's vision. The target becomes visually more prominent in the operative field. Other strong points include simplicity, fair efficacy and safety. In addition, it is relatively new and is; therefore, open to research and development. The expectation is that "fluorescence-guided surgery is here to stay and mature”.

\section{References}

1. Della Puppa A, Ciccarino P, Lombardi G, Rolma G, Cecchin D, Rossetto M. 5-Aminolevulinic acid fluorescence in high grade glioma surgery: surgical outcome, intraoperative findings, and fluorescence patterns. Biomed Res Int 2014; 2014: 232561.

2. Lim C, Vibert E, Azoulay D, et al. Indocyanine green fluorescence imaging in the surgical management of liver cancers: current facts and future implications. J Visc Surg 2014; 151(2): $117-24$.

3. van Dam GM, Themelis G, Crane LM, et al. Intraoperative tumor-specific fluorescence imaging in ovarian cancer by folate receptoralpha targeting: first in-human results. Nat med 2011; 17(10): 1315-9.

4. Guo W, Zhang L, Ji J, Gao W, Liu J, Tong M. Breast cancer sentinel lymph node mapping using near-infrared guided indocyanine green in comparison with blue dye. Tumour Biol 2014; 35(4): 3073-8.

5. Buchs NC, Pugin F, Azagury DE, et al. Real-time near-infrared fluorescent cholangiography could shorten operative time during robotic single-site cholecystectomy. Surg Endosc 2013; 27(10): 3897-901.

6. Dip FD, Asbun D, Rosales-Velderrain A, et al. Cost analysis and effectiveness comparing the 
routine use of intraoperative fluorescent cholangiography with fluoroscopic cholangiogram in patients undergoing laparoscopic cholecystectomy. Surg Endosc 2014; 28(6): 1838-43.

7. Mohsen A, Elbasiouny MS, El-Shazli M, Azmy O, Amr A. Evaluation of the Effectiveness of Fluorescent Visualization of Bile Ducts Using Fluorescein and Ultraviolet A at Laparoscopic Cholecystectomy. Surg Innov 2015; pii: 1553350615610652.
8. Jafari MD, Lee KH, Halabi WJ, et al. The use of indocyanine green fluorescence to assess anastomotic perfusion during robotic assisted laparoscopic rectal surgery. Surg Endosc 2013; 27(8): 3003-8.

9. Ris F, Hompes R, Cunningham C, et al. Nearinfrared (NIR) perfusion angiography in minimally invasive colorectal surgery. Surg Endosc 2014; 28(7): 2221-6. 